\title{
LA OCUPACIÓN COMO ELEMENTO TERAPÉUTICO DE LA PRÁCTICA DE TERAPIA OCUPACIONAL EN PERSONAS CON DISFUNCIÓN FÍSICA
}

\author{
THE OCCUPATION AS A THERAPEUTIC ELEMENT OF THE PRACTICE OF \\ OCCUPATIONAL THERAPY IN PEOPLE WITH PHYSICAL DYSFUNCTION
}

\section{Óscar Hernández Lanas', Erna Navarrete Salas²}

\begin{abstract}
RESUMEN
El presente ensayo aborda el uso terapéutico que los/Las terapeutas ocupacionales entregan a la ocupación, desde elámbito de la rehabilitación en salud física.

El concepto de la ocupación se fundamenta desde una visión histórica y se contrasta con la conceptualización que puedan ser aplicadas al quehacer de terapeutas ocupacionales que trabajan en este ámbito.

A lo largo del escrito se aborda el impacto que tiene la disfunción física en la ejecución de ocupaciones significativa en personas que padecen de esta condición. Se argumenta a favor de mantener la ocupación como núcleo de la intervención terapéutica y la utilidad que presenta como recurso de intervención profesional. Los conceptos de ocupación como medio y ocupación como fin se proponen como una solución práctica para desarrollar intervenciones en este ámbito.

Se concluye que es necesario que los/as terapeutas ocupacionales sean conscientes de la relevancia que tiene el uso de la ocupación al interior de las dinámicas de intervención en salud física; consideren su integración en la practica profesional y expliciten su uso en los procesos de atención directa de personas con disfunción física.
\end{abstract}

\section{PALABRAS CLAVES}

Terapia Ocupacional, Ocupación, Personas con Discapacidad, Rehabilitación.

1 Terapeuta Ocupacional, Magister en Ocupación y Terapia Ocupacional, Licenciado en Ciencia de la Ocupación Humana. Terapeuta Ocupacional Hospital Dipreca. Académico Instructor, Departamento de Terapia Ocupacional y Ciencia de la Ocupación, Universidad de Chile. Independencia 1027, Santiago.+5622951 7275, oscarhernandezlanas@gmail.com.

2 Terapeuta Ocupacional, Master in Medical Sciences in Occupational Therapy, Licenciada en Ciencias de la Ocupación Humana. Profesor Asociada, Departamento de Terapia Ocupacional y Ciencia de la Ocupación, Universidad de Chile. Independencia 1027, Santiago. +5629786587, enavarre@med.uchile.cl. 


\begin{abstract}
The following essay addresses the therapeutic use that occupational therapists give to the occupation, from the field of physical health rehabilitation. The concept of occupation is based on a historical vision and is contrasted with the conceptualization that can be applied to the work of occupational therapists working in this field.

Throughout the paper, the impact of physical dysfunction on the execution of significant occupations in people suffering from this condition is addressed. It is argued in favor of maintaining the occupation as the core of the therapeutic intervention and the utility it presents as a resource for professional intervention. The concepts of occupation as a means and occupation as an end are proposed as a practical solution to develop interventions in this area.

It is concluded that it is necessary that occupational therapists be aware of the relevance of the use of the occupation within the dynamics of physical health intervention; Consider their integration into professional practice and explain their use in the processes of direct care of people with physical dysfunction.
\end{abstract}

\title{
KEYWORDS
}

Occupational Therapy, Occupation, People with Disabilities, Rehabilitation.

Recibido: 4 Diciembre 2018

Aceptado: 2 Diciembre 2019

\section{INTRODUCCIÓN}

Desde el ámbito de atención de terapia ocupacional en salud física, la persona es considerada como el agente que a través de su voluntad puede generar patrones de movimiento, con los cuales logra configurar secuencias espacio-temporales que permiten el desplazamiento de su cuerpo y con ello concretar con libertad la realización de diversas ocupaciones como son las actividades de la vida diaria básicas, instrumentales, tiempo libre, productivas y de participación social, involucrando mente, cuerpo y espíritu, permitiendo un sentimiento de bienestar consigo mismo y con el ambiente.

Como terapeutas ocupacionales, uno de los objetivos de intervención, es lograr este sentimiento de bienestar a través del uso de las ocupaciones, las cuales podemos definirlas como las cosas que las personas hacen todos los días, que tienen un propósito, significado y son culturalmente relevantes (Christiansen \& Townsend, 2004).

Mediante este hacer, las personas desarrollan habilidades, satisfacen necesidades básicas e intrínsecas de dominio y competencia en el ambiente, además de ser un motor importante en el desarrollo de la identidad personal y el logro de la aceptación en el grupo.

En el caso de la intervención en salud física, los/las terapeutas ocupacionales deben estudiar la ocupación de las personas que presentan una movilidad dañada, restringida o limitada por diversos factores, ya sean intrínsecos o extrínsecos, con el fin de entender como esta es impactada en su cotidiano.

Estas personas, muchas veces, manifiestan restricciones o disfunciones ocupacionales, por lo cual como terapeutas ocupacionales debemos identificar las barreras que limitan su participación en las ocupaciones, como la adaptación de las mismas, lo cual será un paso importante en su rehabilitación, para convertirse en un ser activo y próspero (Tabor, Wolf, et al. 2014).

Con estas ideas como punto de partida, el presente ensayo expondrá desde una mirada reflexiva cómo la ocupación contribuye en la intervención de Terapia Ocupacional desde el ámbito de la rehabilitación en salud física, siendo un elemento terapéutico para la práctica en esta área.

\section{Impacto ocupacional de la disfunción física}

La palabra "disfunción" es utilizada en contextos de atención en salud física para indicar una incapacidad crónica o temporal para manejar y participar en los roles, relaciones y ocupaciones esperadas de una persona de similar edad y cultura (Jonsson, Borell, \& Sadlo, 2003); mientras que el concepto de "disfunción 
física" hace referencia a la incapacidad que se produce al tener una enfermedad de origen físico, muchas veces adquirida durante el ciclo vital, que afecta la participación ocupacional esperada de la persona que cursa con esta condición.

Desde la perspectiva de Terapia Ocupacional, la presencia de una disfunción física genera un cambio en la vida de la persona y la obliga a vivir de manera diferente su proyecto vital. Según Rueda \& Aguado (2003), esta vivencia afecta todos los aspectos de la vida, principalmente las capacidades físicas de la persona, limitando su desempeño ocupacional en su vida diaria y en especial, las ocupaciones que considera significativas; debiendo movilizar recursos y estrategias que permitan la adaptación a la nueva situación de salud.

Por su parte, Polonio (2016) señala que la adaptación del adulto a una disfunción física adquirida es un proceso complejo, prolongado en el tiempo y dificultoso, el cual requiere que la persona afectada ponga en marcha sus capacidades cognitivas, emocionales y volitivas para superar su situación actual.

Desde estas perspectivas, podríamos entender que el proceso de rehabilitación en el área de salud física debería englobar no sólo los aspectos físicos, sino también considerar los aspectos psicológicos y socioculturales de la persona, como también los aspectos ocupacionales. La adaptación a esta condición o a su nueva realidad, requerirá el ajuste de sus habilidades, la transformación de sus hábitos y del hacer que tiene esta persona; donde el elemento terapéutico que puede permitir estos cambios es claramente el uso terapéutico de la ocupación.

\section{Uso de la ocupación en los modelos de atención en salud física}

Según Pizzi \& Richards (2017), los modelos de atención en salud física deben velar por obtener el óptimo funcionamiento fisiológico del organismo; componente que se relaciona directamente sobre el cuerpo y la capacidad material del hacer; junto con apuntar a obtener el nivel más alto de salud (dentro de las limitaciones del mismo cuerpo) que permita el funcionamiento óptimo de la persona y con ello obtener el máximo estado de bienestar.
Si tomamos esta propuesta como eje de intervención en salud física, el objetivo de Terapia Ocupacional sería capacitar a las personas para participar en las actividades cotidianas, lo cual, desde un contexto de rehabilitación física, se logra mediante la habilitación de los individuos para realizar aquellas tareas que optimizan su capacidad para participar, o mediante la modificación del entorno que permita o refuerce su participación.

Dentro de las críticas que se realizan a este modelo, Simó (2015) menciona que es un planteamiento restrictivo, que se centra más en el problema de salud mismo y en la recuperación de la enfermedad como la adaptación a la discapacidad, más que en considerar la ocupación como un factor de promoción de la salud y el bienestar. Otros autores que también critican esta propuesta son Morrison, Olivares, \& Vidal (2011) quienes señalan que la ocupación como elemento identitario no aparece en las prácticas cotidianas en salud física, más bien se visualizan intervenciones reduccionistas, centradas en el uso de marcos de referencias y técnicas enfocadas a los componentes neuro-músculos-esqueléticos, centrados en una constante preocupación hacia el cambio de componentes neurológicos, musculares, óseos, emocionales y del comportamiento, configurándolos como el centro de atención en salud física, tanto a nivel preventivo como rehabilitador.

Quizás estas críticas se pueden comprender, si analizamos el desarrollo histórico que ha tenido la terapia ocupacional en el ámbito de la atención de personas con disfunción física. A continuación, se intentarán exponer los fundamentos sobre el uso de la ocupación como elemento terapéutico en la acción de la terapia ocupacional hacia personas con disfunciones físicas, apoyándose principalmente desde un enfoque histórico como teórico.

Según Trombly (1995), desde sus inicios, la Terapia Ocupacional en salud física ha desarrollado abordajes y conceptualizaciones para dar respuesta a diversas problemáticas que presentan estas personas. Dentro del quehacer clásico en esta área, se han utilizado conceptos como la actividad con propósito, el entrenamiento en tareas y por sobre todo el uso de la ocupación como medio y como fin.

Según Morrison \& Vidal (2009), durante el periodo de las ideas conducentes a la fundación de terapia 
ocupacional y la construcción de los primeros postulados y principios, el/la terapeuta ocupacional que trabajaba con población portadora de disfunciones físicas utilizaba como elemento terapéutico las artesanías o ergoterapias, las cuales se fundamentaban por su valor como agente de recuperación de la enfermedad, como también por su valor utilitario, ya que al generar un producto este podía ser vendido.

En esa época, el objetivo central era mantener a la persona ocupada, cambiar sus pensamientos y estar inmerso en algo productivo. El valor terapéutico estaba en generar sentimientos de interés y orgullo personal, favoreciendo la creatividad, la expresión personal, la salud física y mental, enfocando el trabajo desde una perspectiva netamente saludable y en conjunto con otros.

Con el tiempo y con ingreso al mundo hospitalario, la terapia ocupacional se configura como una disciplina de colaboración médica, transitando de la atención centrada en la creación y el hacer a una prescripción para corregir el deterioro atribuible a una enfermedad o lesión corporal (Crepeau et al, 2008; Kielhofner, 2006; Romero \& Moruno, 2003; Turner et al, 2003).

Es durante esta época que las actividades con propósito, mecanoterapias y adaptaciones pasan a ser los elementos terapéuticos centrales del hacer del terapeuta ocupacional. A las personas que debían ser atendidas por terapeutas ocupacionales, se les daba a elegir entre varias actividades, las cuales exigían respuestas similares (patrón de movimiento), instaurando una dinámica de entrenamiento que mejoraba sus capacidades, además de involucrar elementos de su interés. Estas actividades se tornaban significativas y mantenían a la persona interesada en su trabajo. El principal fundamento para estas acciones, fueron las observaciones estructuradas, la reflexión y las experiencias de los primeros terapeutas ocupacionales.

Junto con ello, el/la terapeuta ocupacional adapta las actividades para cambiar las demandas, con la finalidad de involucrar a las personas en su realización y lograr cambiar su desempeño. Además, el enfoque de rehabilitación se va consolidando, y requería que la persona fomentara sus capacidades y preparara su regreso en plenitud a las actividades de la vida diaria y a las pre-vocacionales en relación a la adquisición de un futuro empleo.
Es aquí donde la ocupación se considera como sinónimo de actividades artesanales y/o mecánicas, que se emplean en función de la reparación de un componente del cuerpo o mente que se consideraba deteriorado. La ocupación sería una medicina, para el alivio de los defectos del cuerpo o la mente, mientras que el contexto impone que el ser humano debe realizar acciones para recuperar los componentes deteriorados.

Posteriormente, ya inmersa en el mundo médico, la terapia ocupacional busca fundamentos para dar respuesta a su accionar a través de un enfoque neuromusculoesquelético (Morrison \& Vidal, 2009). Durante esta época, la ortótica y las ayudas técnicas forman parte del hacer cotidiano de los/las terapeutas ocupacionales que se desempeñaban en la atención de problemáticas de salud física.

Es en este período que la ocupación se visualiza como una actividad previamente diseñada y elaborada en función del déficit específico que presenta el ser humano. Mediante las ocupaciones, se logra estimular el cuerpo, para permitir su funcionalidad normal, pueden ser analizadas, diseccionadas en pasos y secuencias, estandarizadas y orientadas al logro de objetivos específicos impuestos por el / la terapeuta ocupacional. Desde este punto de vista, el problema ocupacional sería resultado de una lesión neurológica, musculary/o esquelética, que produce un deterioro o interferencia en la funcionalidad normal del ser humano.

Tal como señala Morrison \& Vidal (2009), este reduccionismo, en su accionar, provoca que los terapeutas ocupacionales reflexionen en torno al concepto de ocupación para así aplicarlo a sus intervenciones, instaurando el periodo conocido como el resurgir de la ocupación.

Nelson \& Jepson-Thomas (2003) señalan que en esta época la ocupación es entendida como una relación de tres variables: la forma, la función y significado. La forma ocupacional es la manera y el modo en que el ambiente estructura el cómo se debe realizar una ocupación. Estas formas son creadas, designadas, estructuradas, diseñadas en la interacción de los seres humanos y el ambiente. El ambiente enseña, modificando o no, la realización de una forma ocupacional. El significado para el ser humano lo entrega el ambiente por medio de la realización y ejecución de las formas ocupacionales. Desde este punto de vista, se concibe el problema 
ocupacional cuando el individuo no es capaz de realizar satisfactoriamente una forma ocupacional.

Desde este concepto cualquier actividad voluntaria se considera ocupación, siempre y cuando la forma ocupacional contenga significado. Desde este punto de vista la persona configura el propósito. Según Clark (1993), se considera como ocupación a toda conducta orientada a objetivos relacionados con la vida diaria, trabajo y ocio, incluyendo actividades espirituales y sexuales.

Se define que la unidad básica de ocupación es la actividad (Trombly, 1999). La actividad es entendida como un comportamiento orientado a objetivos específicos, dirigido hacia el desempeño de una tarea. Mientras que la ocupación se considera como la capacidad de hacery es el elemento global para comprender el accionar de la Terapia Ocupacional. Es en esta época que se asume que el gran problema de las personas con disfunción física es el impedimento del hacer o la incapacidad de realizar, situación entendida hoy como disfunción ocupacional.

\section{Ocupación como un elemento terapéutico central de la intervención}

Dependiendo de la época histórica en que se analice el quehacer de la Terapia Ocupacional, se dispone de diferentes respuestas a las problemáticas ocupacionales para sus beneficiarios con disfunción física.

Lo que debe ser claro es que la Terapia Ocupacional en personas con disfunción física debe apoyar al retorno de la ocupación. Tal como menciona Da Silva et. al. (2011), se debería apuntar a una comprensión ocupacional del ser humano, lo que traspasa las fronteras profesionales, posibilitando ampliar las habituales dimensiones de comprensión como ser biopsicosocial a la dimensión ocupacional.

Además, la ocupación debe ser entendida como aqueIla actividad con sentido en la que la persona participa cotidianamente y que puede ser nombrada por la cultura (Álvarez et al., 2007). Esta definición indica una acción, un hacer o un pensar, que conlleva a cierto impacto con el entorno para transformarlo y transformarse. La ocupación no puede ser entendida sin un contexto, ni aislarse de otras actividades, ya que establece relaciones en múltiples dimensiones que hacen que su ocurrencia sea única. Esta ocurrencia hace que la persona le dé sentido, la vincule a su historia, sus creencias y vida dentro de un entorno culturalmente irrenunciable.

Para que la persona desempeñe una ocupación, esta debe referir sentido, entendido como el valor personal que le asigna a las actividades y que se construye desde las experiencias sensoriales, afectivas, motoras, cognitivas, sociales y espirituales. El sentido presenta un componente histórico, donde las experiencias, su efecto y su subjetividad impactan el hacer; el cual puede cambiar, influyendo en la intensidad con que la persona vivencia un hacer particular, o en las elecciones de formas ocupacionales que hace; en concordancia con su identidad, su historia y su pertenencia. Por otro lado, la construcción social y cultural que se da en la ocupación es conocida como significado, el cual da arraigo a su entorno y al igual que el sentido, contribuye a la identidad, historia y pertenencia.

Esta definición muchas veces no se logra vislumbrar de forma explícita en la acción diaria del terapeuta ocupacional que se desempeña en contextos tradicionales de la atención en salud física, como hospitales y centros de rehabilitación física. En estos contextos, existe un nivel elevado de demanda sanitaria, por lo que el profesional debe centrar su atención a factores como la productividad, nivel de eficiencia y los costos de la atención, lo cual pondría en segundo plano el valor de la ocupación en sí misma como elemento terapéutico.

Para ello, desde la intervención de Terapia Ocupacional en salud física se puede utilizar la ocupación en dos formas: como vehículo para conseguir un fin fuera de la propia actividad (ocupación como medio) y como método para hacer que el usuario se involucre en actividades que le permitan mejorar su participación en sus ocupaciones diarias y significativas (ocupación como fin) (Ferguson \& Trombly, 1997; Trombly, 1995).

Al entender la ocupación como medio, es comprenderla como un agente de cambio terapéutico que favorece el desarrollo de las habilidades deterioradas por la disfunción física. Es desde este enfoque que el/la terapeuta selecciona actividades utilizando los componentes de las mismas para generar una respuesta terapéutica deseada, es decir, aumentar las habilidades de la persona. Según Cynkin \& Robinson (1990), para que la ocupación sea un medio terapéutico debe tener propósito, es decir, que exista una meta desafiante, pero 
con posibilidad de éxito, además de tener significado y relevancia para la persona, quien debe motivarse a ejecutarla e involucrarse en ella.

Al entender la ocupación como fin, es darle intencionalidad al hacer, es decir, es dar dirección al hacer hacia algo importante y significativo para la persona con disfunción física. Desde este enfoque se permite organizar el comportamiento, la rutina diaria y en especial la vida de la persona, dado que sólo las ocupaciones significativas permanecen en el repertorio de la vida cotidiana de la persona con disfunción física. Desde esta modalidad de intervención, el rol del terapeuta ocupacional, es motivar y facilitar el hacer o lo que se deja de hacer, es decir es un facilitador, más que un restituidor de habilidades para ejercer la ocupación.

Siguiendo los lineamientos de Wilcock (1998), los/las terapeutas ocupacionales entienden que existe una unidad intrínseca en las personas para estar activos, y participar satisfactoriamente en ocupaciones significativas, dado que el estar ocupado es la esencia de todo lo que hacemos y con ello, la ocupación se configura como la herramienta clave para la promoción de la salud y el bienestar.

\section{CONCLUSIÓN}

Como reflexión final es posible señalar que las personas con disfunción física presentan dificultades variadas que pueden ser intervenidas más allá de la esfera de la misma disfunción. Su proceso de rehabilitación debe ser enmarcado desde un enfoque ocupacional, ya que la utilización de este permite comprender las barreras que limitan la participación de la persona, las estrategias de adaptación utilizadas para afrontar su nueva situación de salud, además de entregar información sobre sus necesidades, potenciales y motivaciones en el hacer.

Junto a ello, es importante recordar que, al utilizar un enfoque ocupacional, no empleamos una mirada restrictiva de la realidad, sino más bien es una invitación a comprender el fenómeno de forma global, permitiendo comprender el problema más allá de la dificultad de salud y los mecanismos de enfermedad.

Utilizar la ocupación como centro de la atención en salud física, requiere ampliar la visión que se ha tenido históricamente sobre el problema, por lo cual es de vital importancia que en este contexto se valore el uso de la ocupación como agente terapéutico.

Desde una visión histórica, el uso de la ocupación en salud física ha sido conceptualizado de diversas formas, pasando de acciones que buscan remediar un déficit a las que poseen un enfoque más integrado y que pretende la inmersión total de la persona en su hacer. Estas conceptualizaciones han ido evolucionando en la disciplina e integrando diversos elementos que enriquecen el uso de la ocupación como elemento primordial en el hacer profesional de los/las terapeutas ocupacionales, destacando en este aspecto el uso de la ocupación como medio o como fin.

Dentro de este punto, el uso de la ocupación como medio es una herramienta que permite mejorar habilidades, facilitando que la persona sea un ser activo que aprende, se adapta, y es capaz de participar en diferentes ocupaciones, mientas que la ocupación como fin se configura como una herramienta que permite recuperar, mantener o prevenir la disfunción física, consintiendo que la ocupación sea utilizada para fines evaluativos, preventivos y terapéuticos.

Resulta interesante considerar estas conceptualizaciones en las intervenciones, ya que permiten apuntar a una visión holística de los problemas que afectan a las personas que presentan una disfunción física. Además, al reconocer y analizar la ocupación, se produce un enfoque único dentro de la terapia ocupacional que ayuda en la planificación de las intervenciones que realizan sus profesionales.

Como idea final, es necesario que como terapeutas ocupacionales seamos conscientes de la relevancia que tiene el uso de la ocupación como elemento central para el abordaje de personas con disfunción física. Por lo cual es importante el manejo de conceptos que sean utilizados en la práctica, y así emplearlos en el proceso de intervención, procurando considerar a la persona como un ser integral y por sobre todo, un ser ocupacional el cual es capaz de participar en diversos contextos y situaciones. 


\section{REFERENCIAS BIBLIOGRÁFICAS}

Álvarez, E., Gómez, S., Muñoz, I., Navarrete, E., Riveros, M., Rueda, L., Valdebenito, A. (2007). Definición y desarrollo del concepto de Ocupación: ensayo sobre la experiencia de construcción teórica desde una identidad local. Revista Chilena de Terapia Ocupacional, 7(1), 76-82. https://doi.org/10.5354/0717-5346.2007.81

Bruner, J. S. (1990). Acts of Meaning. Harvard University Press.

Clark, F. (1993). Occupation embedded in a real life: interweaving occupational science and occupational therapy. 1993 Eleanor Clarke Slagle Lecture. The American Journal of Occupational Therapy: Official Publication ofthe American Occupational Therapy Association, 47(12), 1067-1078.

Christiansen, C, Townsend, E., (2004). Introduction to Occupation: The Art and Science of Living. Upper Saddle River, NJ: Prentice Hall; Maslow AH.

Crepeau, E. Cohn, E. Schell, B.(2008). Willard and Spackman's Occupational Therapy. 11th Edition. Lippincott Williams \& Wilkins.

Cynkin, S., \& Robinson, M. (1990). Occupational therapy and activities health: Toward health through activities. Boston: Linle Brown.

Da Silva Araújo, L., Fierro Jordán, N., Guitérrez Monclus, P., \& Rodríguez, O. (2011). Ciencia de la ocupación y terapia ocupacional: sus relaciones y aplicaciones a la práctica clínica. Revista Chilena de Terapia Ocupacional, 17(1), Pág. 79-87. doi:10.5354/07195346.2011.17084.

Ferguson, J.M., \& Trombly, C. A. (1997). The effect of added-purpose and meaningful occupation on motor learning. American Journal of Occupational Therapy, 57(7), 508-515.

Fleming, M.H. (1991). The therapist with the three-track mind. The American Journal of Occupational Therapy: Official Publication of the American Occupational Therapy Association, 45(11), 1007-1014.

Jonsson, H., Borell, L., \& Sadlo, G. (2003). Jubilación: Una transición ocupacional con consecuencias en la temporalidad, equilibrio y significado de la ocupación. Revista Chilena de Terapia Ocupacional, (3), 1-7.

Kielhofner, G. (2006). Fundamentos Conceptuales de la Terapia Ocupacional ( $3^{\circ}$ edición). Buenos Aires: Médica Panamericana.

Lillo, S. G. (2003). La ocupación y su significado como factor influyente de la identidad personal. Revista Chilena de Terapia Ocupacional, (3), 30-43.

Morrison, R., Olivares, D., \& Vidal, D. (2011). La filosofía de la Ocupación Humana y el paradigma social de la Ocupación. Algunas reflexiones y propuestas sobre epistemologías actuales en Terapia Ocupacional y Ciencias de la Ocupación. Revista Chilena de Terapia Ocupacional, 17(2), 102-119.

Morrison, R., \& Vidal, D. (2009). Análisis ontológicos de la Ocupación Humana (Seminario de Título). Universidad Austral de Chile, Valdivia. Recuperado a partir de http://cybertesis.uach.cl/tesis/ uach/2009/fmv648a/doc/fmv648a.pdf
Nelson, D., \& Jepson-Thomas., J. (2003). Occupational Form, Occupational Performance, and a conceptual framework for Therapeutic Occupation. En: Perspectives in Human Occupation: Participation in life. Philadelphia: Lippincott, Williams, and Wilkins.

Tabor, L., Wolf, T., Foster, E., Hildebrand, M., Baum, C.,(2014) Participation and engagement in occupation in adults with disabilities. En: Pierce, D., Occupational Science for Occupational therapy (p. 107-120) New jersey: Editorial SLACK Incorporated.

Pizzi, M. A., \& Richards, L. G. (2017). Promoting Health, Well-Being, and Quality of Life in Occupational Therapy: A Commitment to a Paradigm Shift for the Next 100 Years. American Journal of Occupational Therapy, 77(4). https://doi.org/10.5014/ajot.2017.028456

Polonio, B. (2016). Terapia Ocupacional en Disfunciones Físicas: Teoría y práctica ( $2^{\circ}$ edición). Buenos Aires: Medica Panamericana.

Romero, D., \& Moruno, P. (2003). Terapia Ocupacional: teoría y técnicas. MASSON, S.A. Barcelona, España.

Rueda, M., \& Aguado, A. (2003). Estrategias de afrontamiento y proceso de adaptación a la lesión medular. Madrid: Instituto de Migraciones y Servicios Sociales.

Simó, S. (2015). Una Terapia Ocupacional desde un paradigmo crítico. Revista de Terapia Ocupacional de Galigia (A Coruña). Monog. 7: 25-40.

Trombly, C. (1995). Occupation: Purposefulness and meaningfulness as therapeutic mechanisms. American Journal of Occupational Therapy, 49(10), 960-972.

Turner, A., Foster, M., \& Johnson S. (2003). Terapia Ocupacional y Disfunción Física. Principios, Técnicas y Práctica. $5^{\circ}$ Ed. El Servir España S.A.

Wilcock, A. (1998). An Occupational Perspective of Health. Thorofare, NJ: Sack Inc

World Federation of Occupational Therapists. (2005). Position Statement Occupational Science. Resource Centre, World Federation of Occupational Therapists. 